\title{
DESIGN SYNTHESES AND ANALYSES OF A LAB ON A CHIP (LOC) MODULE BASED ON BIOLOGICAL CELL REQUIREMENTS IN NATURE
}

\author{
J.A. ALQABANDI \\ Mechanical Engineering Dept., Mechatronics in Medicine Laboratory, \\ Imperial College London, UK
}

\begin{abstract}
It is of a growing interest among researchers in the biotechnology field to acquire a reliable system that maintains the viability of a cell in an in vitro environment for a sufficient period of time, and provides multi-task analyses on a mammalian cell. Therefore, the Lab-on-a Chip (LoC) field has been initiated to address such needs. The objectives of this work are (1) to provide a review on how nature defines the design requirements of a miniaturized system for cell viability - mimicking that of an in vivo domain, as well as extracting cellular electrophysiology at a molecular level, and (2) to translate such requirements into an engineering application of design synthesis and analyses of two main integrated components of an LoC platform: microfluidic $(\mu \mathrm{F})$, and Multi-Electrode-Array (MEA) systems. As a result, this work highlights the optimal environment of a cell to live and grow for bioresearch by acquiring an engineered system of nutrition supply and removal of wastes (perfusion), $\mathrm{pH}$ neutralization, sufficient supply of oxygen, thermal stability, elimination of air pockets, and a presence of a highly salted aqueous solution. On the essence of cellular nature and design interrelation, the results of the Computational Fluid Dynamic (CFD) analyses visualize and predict flow characteristics, investigate the optimization process of having a uniform flow pattern (uniform nutrition distribution), elimination of air pockets for cell within the microfluidic module, and arriving at a stable system in terms of controllability and durability. Similarly, the MEA's empirical analyses define an optimal pitch distance between two neighbouring electrodes that would visualize and arrive at a uniform current density distribution, allowing sufficient space for cell-line growth. In conclusion, the biology principles should be comprehended prior to modelling, design, and microfabrication of a LoC, to place such module as a valuable tool for bio-experimentalists.

Keywords: Biotechnology, cellular electrophysiology, cell viability, constructal law, in vitro, in vivo, lab-on-achip, microfluidic, multi-electrode arrays.
\end{abstract}

\section{INTRODUCTION}

A Lab-on-a Chip (LoC) field, also referred to in literature as a micro-total-analytical system ( $\mu$ TAS), has been initiated to address the requirements of having a miniaturized durable system that preserves biological cells' viability within an in vitro environment, enabling experimental analyses for a sufficient period of time; it is astonishingly motivating to find that despite the infancy of the LoC field commencement date, it has grown massively in a very short time not only capturing biologists' attention, but also that of astrologists as well, for space travel bio-experiments, by addressing constraints within different spectrum of science in a novel manner [1]. For instance, a major constraint faced by bio-researchers is the randomness of signals and noises experienced within a population of cells, which can be eliminated by sorting a single cell in a confined and controlled environment such as that of a microfluidic domain [2].

The reader should value the fact that it is crucially important to comprehend the biology principle, merits of LoC, electrophysiology, and mechanobiology of cells, prior to modelling, design and fabrication of bio-mechatronic platform systems-in order to arrive at the biological consequences and impacts on design aspects of an LoC domain integrated within the bio-mechatronic platform. For example, some cells exhibit adhesive properties that can be considered as an added advantage in terms of fixation of the cell on a LoC surface for lab analyses, yet it might lead to clogging and poor

(C) 2015 WIT Press, www.witpress.com

ISSN: 1755-7437 (paper format), ISSN: 1755-7445 (online), http://www.witpress.com/journals

DOI: 10.2495/DNE-V10-N1-70-86 
controllability of cell manipulation [2]. At the same time, disengaging cells from their adhesive state, could damage the cell membrane and lead to random signal propagations [3].

This work throws light on nature, e.g. biology, as a protagonist that defines, sets design parameters, and influences the associated synthesis and analysis processes within the engineering field. Biotechnological miniaturized devices are just an illustration of how various elements of flow system components (e.g. brain neurons, immune cells, alveoli within lungs, etc.) are livingly active with respect to each other in a background of a flow defining what the follow should carry (e.g. electrochemical signals, mechanical signals, etc.), and where each component of the flow system should be optimally located to ensure durability and high level performance over time [4, 5]. In line of this concept, the miniaturized platform that shall be further addressed in this study, e.g. array of microelectrodes patterned on a glass substrate should work hand in hand with another miniaturized component (microfluidic system) within the platform (the overall flow system) on the bases that the former flow system component's design (configurations and generated drawings prior to microfabrication) ensures faithful extraction of cellular electrochemical signals within a uniformly distributed electric current in a damage-free cell environment within a contained microfluidic system; such anticipated flow system should preserve cell's integrity throughout the conducted experiment time frame. Therefore, the importance of flow systems (non-equilibrium systems) lies within the resultant efficient design that ensures durability and optimal utilization of any miniaturized system over time, shortens lead time by avoiding trial and error, and further enhances system components' performance; this is what defines the Constructal theory/law: 'For a finite-size flow system to persist in time (to live), it must evolve in such a way that it provides greater access to the currents that flow through it' [4]. Driven by the two great works in [4, 5], advocating Constructal law in justifying, in particular, the evolution of miniaturized devices on the essence of biology, and presenting design as science rather than art in assembling and optimizing a constructed platform, shall further assist in defining design boundaries as well as limitations (imperfections), where sources of enhancements could be allocated.

This work illustrates nature's role in defining design parameters of a miniaturized system for cell viability within an in vitro domain capturing that of in vivo, and extracting cellular biophysics at a molecular level. As a result, the identified design boundaries are transformed into engineering applications of design synthesis and empirical analyses-setting the design parameters of two integrated components of a LoC platform: microfluidic $(\mu \mathrm{F})$, and Multi-Electrode Arrays (MEAs). The optimal environment of a mammalian cell to live and grow for bioresearch, by acquiring an engineered system of nutrition supply and removal of wastes (perfusion) and carbon dioxide, $\mathrm{pH}$ neutralization, sufficient supply of oxygen, thermal stability, elimination of air pockets, and a presence of a highly salted aqueous solution are underlined and further discussed within this paper.

\section{INTEGRATED MICROFLUIDIC (I $\mu$ F) SYSTEM}

\subsection{Design synthesis}

Microfluidics $(\mu \mathrm{Fs})$, avail researchers the benefits of analysing a single or a population of cells in a confined and controlled environment with a very high sensitivity [6-8]. Fragoso et al. [8] have fabricated an $i \mu \mathrm{F}$ platform to detect breast-cancer-protein markers in a serum medium, based on its electrochemical (electrophysiology) characteristics. They have designed their system to house two zones: (1) detection with $\mu \mathrm{F}$ chambers and their associated 16 working electrodes, and (2) fluidic storage zone of a cell sample and reagents consisting of five reservoirs. 


\subsubsection{System perfusion}

In mammalian bodies, perfusion is the process in which nutrients, oxygen and cell growth factors are provided to cells, and wastes such as carbon dioxide $(\mathrm{C}$,) insoluble cell debris are removed from the system. Perfusion can be branched into (1) re-circulating (for large volume media: re-circulated through the system where wastes are diluted and sent back to cells), and (2) non-re-circulating (media is perfused into the system and then sent entirely to waste) [9]; perfusion is an essential process of supplying nutrients to cells. Figure 1 shows microfluidic perfusion system [10].

Kim et al. [11] have designed and fabricated a continuous perfused $\mu \mathrm{F}$ domain with a system of 3- and 4-way valves to culture murine adhesive embryonic stem cells (ESCs) for four days until cell confluence (cellular dense culture) has been reached. Their main objective was to model and design a variable flow rate initiated from a single input. They have noticed that slow flow rate environment didn't experience cell growth as opposed to a constant higher flow rate, where cells have grown in a healthy-round-morphology shape. In the absence of neighbouring cells, they have estimated the shear stress exerted on a single cell [cell's height $(k)$ ], adhered to a channel of height $h$, to be three times the fluid shear stress experienced at walls. However, they have concluded that shear stress has a minimal effect on the morphology of cell colonies, and that high flow rate plays an important role in terms of expediting the process of waste removal, nutrient delivery and removal of proliferation (growth factor) concentration. Furthermore, continuous perfusion ensures non-evaporation of the contained fluid volume within the system and multi-perfusion channels prevent the cell from being flushed away, and ensure uniform nutrient distribution [10].

Nevill et al. [12], on the other hand, have developed an on-chip-i $\mu \mathrm{F}$ system of a continuous perfusion cell culture and 'on-demand-cell lysis'. They have cultured HeLa, MCF-7, Jurkat, and CHO-K1 cell in a 5-day-time period, and they have managed to lysing (breaking) cells in a lysing-reagent-free environment. In their device, the lysis process is achieved by imposing a DC potential that leads to the development of hydroxide within the proposed system, without the need for higher electric field development. The electrochemical cell lysis [13], on the other hand, is carried out by inducing a DC potential through an electrode neighbouring the trapped cell, where hydroxide is initiated at the cathode upstream. In [13], $\mathrm{Cr} / \mathrm{Au}$ as opposed to Ti/Pt configuration has been considered in their designed experiment as a robust electrode model.

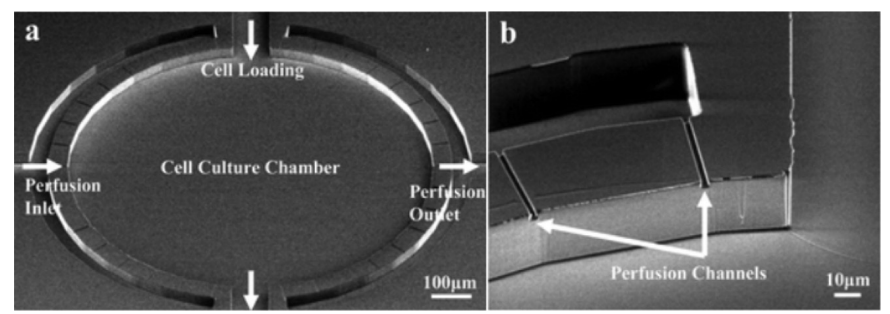

Figure 1: A micrograph of 1-mm-diameter-culturing reservoir with perfusion channels prior to adherent to a glass substrate. High aspect ratio design. (a) SEM picture of a single unit of the arrayed device before bonding. Multiple perfusion channels surround the main culture chamber. The microchamber is $40 \mu \mathrm{m}$ in height with a diameter of $1 \mathrm{~mm}$. Each culture unit has four fluidic access paths. (b) SEM image of perfusion channel dimensions. Each perfusion channel had a width of $2 \mu \mathrm{m}$ and height of $2 \mu \mathrm{m}$-Reproduced with text caption from [10] with permission from the Royal Society of Chemistry. 
Hung et al. [10] have designed and fabricated a novel microenvironment for high throughput Human Carcinoam (HeLa) cell culture consisting of a circular $\mu \mathrm{F}$ chamber enclosed by a number of perfusion channels-cell culturing lasts for 8 days. The culturing was performed at $\mathrm{C}$, until cell confluency has been achieved. It is shown in [10] that high aspect ratio of the $\mu \mathrm{F}$ chamber to the multi-perfusion channels leads to a large fluidic resistance, which eventually contributes into having a uniform flow pattern (uniform nutrition distribution) for cell. Similarly, Kim et al. [9] highlight the importance of optimal controllability on perfusion process: supply of nutrients and removal of wastes of adhesive cells.

\subsubsection{Mimicking in vivo environment}

Prior to investigating the relevant literature on simulating in vivo domain within the in vitro environment, it is substantially worth drawing a comparison and highlighting the merits associated with $\mu \mathrm{F}$ system in the in vitro environment to those of static Petri dish culturing, as well as humanized/nude mouse models [14, 15], where the latter are utilized in the in vivo model within biomedical research. $\mu$ Fs provide high resolution and throughput in obtained measurements, realtime monitoring of the system, spatial and temporal controllability over the $\mu \mathrm{F}$ domain $[16,17] . \mu \mathrm{F}$ based-LoC reduces the usage of lysing reagents [12]. On a less account than static petri-dish culturing, LoCs exhibit less evaporation rate and temperature variability. Their designed system allows the analyses of capillary morphogenesis as in tumour growth and wound healing [16]. On the other hand, static petri-dish-culturing approach experiences uncontrolled signalling, and accumulation of cell's waste. In addition, static culturing doesn't provide full spatial controllability over cell movements [18]. $\mu$ Fs provide a variety of flow gradient that allows performance of complex bioexperiments, enabling prompt switching (continuous flow) or inclusion of cell-cell communication (no flow) [18].

$\mathrm{I} \mu \mathrm{F}-$ based LoC module can suppress the costly robotic cell-culturing platform, especially in the case where a macro-level robot cannot conduct culturing at a miniaturized scale [19]. Also, i $\mu$ Fs have a number of merits over static culturing by controlling the sheer stress, which is advantageous for some types of cells, e.g. vein endothelial [20, 21]; also, controlling shear stress enhances perfusion process once the system geometries are optimized [20].

$\mu$ Fs operates on a small culturing volume: providing most beneficial experimental environment, and hence greatly eliminating labour intensive factor, and lessening the occurrence of experimental systematic errors [19]. It is reported in [22] that $\mu \mathrm{F}$ approach provides more sensitive analyses, and less utilized reagent volume than static culturing. Moreover, cross contamination is less likely to take place during $\mu \mathrm{F}$ experiments as opposed to static culturing processes.

In vivo mouse model approach is a process of nurturing tumours within mammalian bodies of animals such as mice $[14,15]$; it is a very time consuming and tedious process that suffers from lack of legitimate model to perform the experiment. Also, it is quite expensive, containing lots of overheads to maintain the in vivo model (i.e. animal technician would charge $\sim$ US $\$ 100 /$ per day for mouse maintenance). Also, there is the issue of patenting and obtaining permission of utilizing a patented developed mouse model, as well as the monotonous/lengthy culturing procedures, and ethical obligations that the bio-experimentalists have to adhere by. Moreover, it acquires a lengthy lead time in obtaining approvals for certified protocols when conducting the mouse model experiment. Thus, $\mu \mathrm{F}$ approach could provide a simulated in vivo model within the in vitro environment, without all suffer that a laboratory animal is enduring.

In line of the merits of $\mu \mathrm{F}$ chamber over static culturing process, further research is geared towards mimicking in vivo that in particular should benefit tissue engineering field. Zilkowska et al. [20] have provided a detailed review of utilizing $\mu \mathrm{F}$ to mimic in vivo environment. They have stated that 
a resemblance of in vitro to in vivo environment leads to a realistic cell growth, but on a slower rate than static culturing. Vickerman et al. [16] have developed a novel multi-parameter control of Polydimethylsiloxane (PDMS) $\mu \mathrm{F}$ platform, via soft lithography, for a 3D-cell culturing in synthetic hydrogel, powered by imaging processing module. This cell-scaffolding technique, of known pore size, density, and stiffness consisting of various cell types in a designed spatial arrangement, simulates tissue engineering in the in vivo environment. As shown in Fig. 2, their proposed system consists of microfluidic channels with a central gel cage. In their work, gel cage micro-pillar array is utilized to provide rigid platform when seeding cells into scaffold. It should be noted that during perfusion flow, mechanical stresses are imposed on the 3D matrix cells entrapped in gel that requires a design for rigidity by embedding nano-pillars. The hydrogel scaffold is microinjected in a pre-set protocol with or without cell into the gel cage [16].

Kim et al. [23] have also developed a 3D-cell-culturing platform for differentiating embryonic stem cells (ESCs) in an embryoid body. 3D culturing is more efficient within pharmaceutical industry in reporting results of drug screening than 2D culturing, as it provides cell-cell mechanical and extracellular matrix (ECM) interaction resembling that of an in vivo tissue [23]. Cell interference with its in-range vicinity is normally accomplished by adhesion to another cell, and through ECM via its surface macromoleculars [24]. Alternatively, Leclerc et al. [25] have uniquely designed and fabricated a 3D-PDMS- $\mu \mathrm{F}$ device of two stacked layers for Hepatocarcinoma liver (Hep G2) cell culture for eight days until confluence has been reached. They have identified that glucose consumption and albumin production are a manifestation of ongoing activity of viability of cells. To maintain viability of cultured cells, the culture medium has to be altered routinely to provide glucose (nutrients) on a 2-day basis to maintain cell viability [25]. Distinctively, they have estimated the oxygen consumption within the $\mu \mathrm{F}$ device based on the product of cell's density, area of cell culture, and cell oxygen consumption per second; also they have illustrated that the permeability of oxygen (induced to the system) through PDMS compartment is based on the product of permeability of PDMs into the ratio of the oxygen gradient concentration to the thickness of PDMS walls. However, there are some constraints associated with PDMS permeability, where water vapour and organic solvent could enter the PDMS through its permeability feature [9]. Thus, for better experimental productivity, PDMS-based $\mu \mathrm{F}$ device should be placed in a vacuum chamber to remove all moisture prior to conducting an experiment.

Cheng et al. [26] have utilized a biocompatible 3D E-coli cell assembly within a $\mu \mathrm{F}$ domain with parallel sidewall $\mathrm{Cr} / \mathrm{Au}$ electrodes, via spatially programmable gel formation, and controlled $\mathrm{pH}$ parameter. They have utilized calcium-responsive alginate hydrogel, which they have electrochemically deposited into the $\mu \mathrm{F}$ platform. Here, they've uniquely defined side-wall electrodes, enabling trapping cell feature within a hydrogel, where cells are adhered to the calcium alginate gel on the anode for a $3 \mathrm{D}$ visualization of the cell growth on the gel substrate.

\subsubsection{Thermal stability and $\mathrm{pH}$ neutralization}

The $\mu \mathrm{F}$ domain experiences high surface to volume ratio, which should increase diffusion rate and heat conduction processes [7]. Therefore, high surface to volume ratio alarms for temperature built up, and hence designers should carefully investigate the approaches in thermally stabilizing the system [18]. Variability in temperature affects viscosity of the medium, and thus the overall velocity profile of the flow [27]. Many researchers maintain temperature stability by incorporating transparent indium-tin-oxide (ITO) heaters into their $\mu \mathrm{F}$ platform $[8,10]$. It is reported in literature that stable temperature and $\mathrm{pH}$ rate should respectively be $\mathrm{C}$, and $7.2-7.4$ within $5 \% \mathrm{C}$ [9]. Oscillation rate, entrapped in a $\mu \mathrm{F}$ chamber, is interrelated to $\mathrm{pH}$ acidity within the system that affects any extracted electrophysiology characteristics out of a bio-system [28]. 


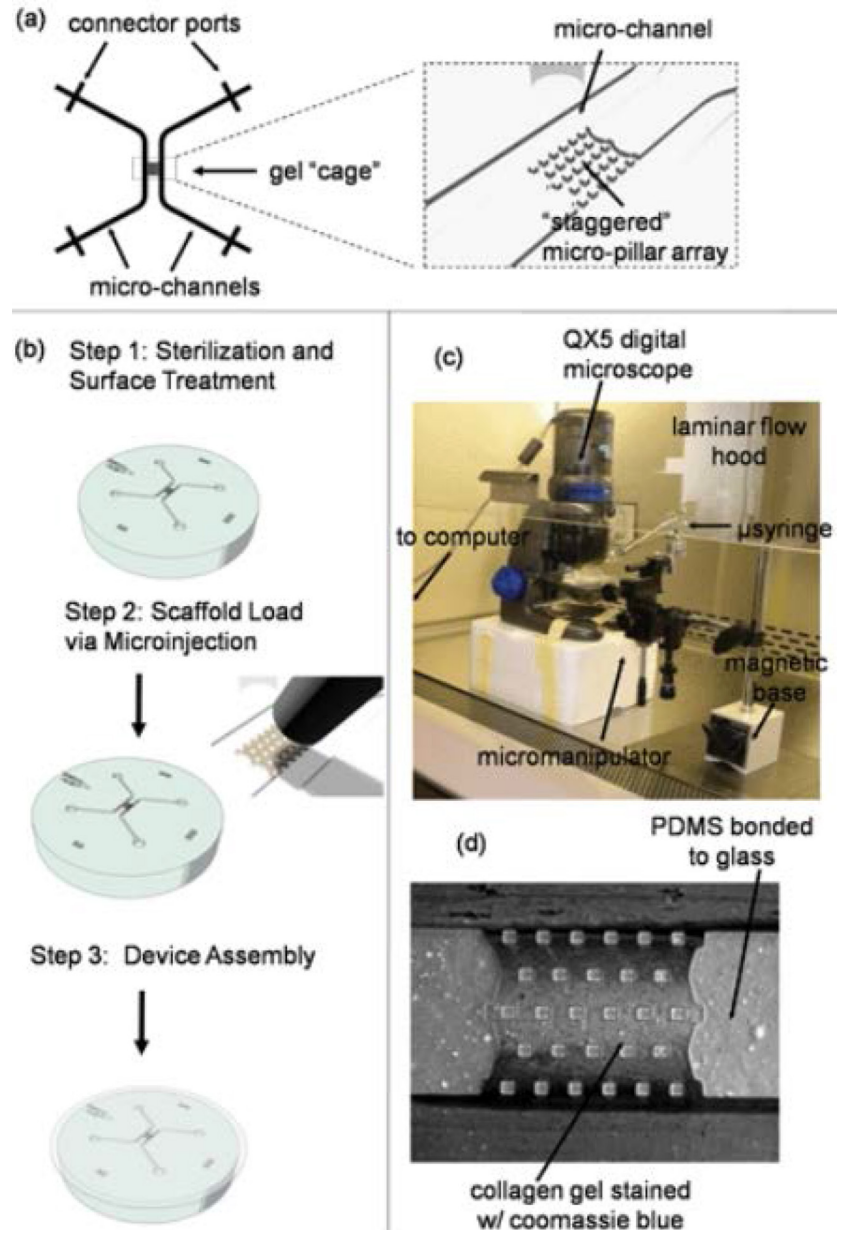

Figure 2: A 3D matrix of central gel cage for cell culturing illustrating in vivo tissue engineering. Schematic of $\mu \mathrm{FD}$ and scaffold loading protocol. (a) The $\mu \mathrm{FD}$ consists of two parallel channels for controlling fluid flow and a central gel cage for 3D cell culture. One key feature of the gel cage is the staggered array of micro-pillars, which provide mechanical support for soft hydrogels. (b) Scaffold loading protocol. PDMS wafers were fabricated. PDMS wafers were cleaned, sterilized and surface treated (Step 1). Pre-polymer solution was precisely microinjected in the gel cage. Hydrophilic PDMS surface permitted proper gel spreading and filling (Step 2). Microfluidic channels were defined by sealing device with glass cover slip. (c) Microinjection station in sterile laminar flow cell culture hood. Precise scaffold loading can be achieved with a custom-made microinjection setup which includes digital microscope, and xyz micromanipulator with microliter syringe. (d) Micrograph of assembled $\mu$ FD showing media channels and collagen partially stained with coomassie blue. Both biologically derived and synthetic matrices used for traditional macro-scale 3D cell culture can be precisely immobilized in gel cage. With the gel in place media channels are virtually isolated except for diffusive or convective transport of molecules through the porous matrix-Reproduced with text caption from [16], with kind permission of Prof. Roger Kamm, Biological/Mechanical Engineering Dept., Massachusetts Institute of Technology (MIT), and with permission from the Royal Society of Chemistry. 
Eddington et al. [29] have developed an $\mathrm{i} \mu \mathrm{F}$ system, in which the $\mathrm{pH}$ is automatically controlled in a self-regulating mechanism of an input stream with respect to the output. In [30], a label-free, low-cost, and disposable polycrystalline silicon TFT have been utilized as a biological sensor of specific ions and analytes that could be extended to monitor $\mathrm{pH}$ concentration. It is demonstrated in [10] that after cells' settlements in the bottom of the $\mu \mathrm{F}$ chamber, $\mathrm{C}$ independent medium was flown through the perfusion channels; during the continuous perfusion, the whole system is placed into an incubator to control humidity and temperature, where is permitted through the permeability feature of PDMS and continuous feeding of perfused nutrition ensured a stabilized $\mathrm{pH}$ rate [10]; incubators are used to maintain cultured medium in a $5 \% \mathrm{C}$ rate.

Dimov et al. [13] have illustrated a novel $\mathrm{i} \mu \mathrm{F}$ array plate (iMAP) for gene expression, protein immunoassay, and cytotoxicity of real-time optical molecular analyses of adhesive cells (HeLa, MCF7), and non-adhesive cells (Plasma cells U266, macrophage J774). Their developed $\mu$ F system consists of two-layer soft lithography with no requirement of alignment. By maintaining a channel to trench depth ratio greater than 5 with an inlet fluid velocity of $100 \mu \mathrm{m} / \mathrm{s}$, they have successfully managed to trace a particle with $100 \%$ efficiency in their obtained simulation. They harness the importance of analysing a biological molecular, such as those of circulating tumour cells (CTCs) nature, which have a very seldom frequent occurrence to be captured during microculture analyses.

\subsubsection{Elimination of air pockets}

Bubbles could lead to clogging (malfunction) of the $\mu \mathrm{F}$ system. A non-uniform flow leads to bubble formation and cell death [25]. Elimination of air pockets could be accomplished in different fashions. For example, from a practical experience, removal of air bubbles within a $\mu \mathrm{F}$ device is performed by filling the domain with DI water on a low pressure for about 30 minutes: not to break the bonding between the $\mu \mathrm{F}$ and glass substrate. Also, Phosphate Buffered Saline (PBS) flushing can be performed to ensure a bubble-free environment prior to cell culturing as in [12]. Furthermore, a bubble trap could be installed interconnecting the syringe pump and first inlet valve. Vacuum chamber is utilized during fabrication for surface treatment, and removal of air bubbles. Also, a bubble tank is incorporated between culture medium tank, and the $\mu \mathrm{F}$-peristaltic pump platform for a system in a loop. During sterilization, ethanol also removes air bubble. From an electrode perspective, increasing potential may contribute into formation of air bubbles, and reducing the flow rate, which also could contribute into improper supply of nutrients to the cell as well as a generation of backflow.

Bubble accumulations on an electrode can degrade its performance by deducing its emitted current. Channels with slit, pit, and/or groove features and/or with sharp corners are source of air-bubble-pocket formation [31]. Electric means, capillary force, ethanol can all be utilized to eliminate such bubble accumulation if they are well controlled.

\subsubsection{Sufficient supply of oxygen}

Oxygen supply is dominantly indispensable for cells to maintain their viability. The mammalian cell metabolism mechanism is dependent on supply of oxygen and removal of carbon dioxide [18]. Thus, the system has to mimic the balance between supply and demand achieved in metabolism. However, cells' consumption of oxygen varies from one type to another. As an example, embryonic stem cells require low consumption rate for better proliferation [32], whereas hepatocytes demand high oxygen consumption rate $[33,34]$. As the cell density increases, the oxygen consumption proportionally increases [18].

The vast majority of $\mu \mathrm{F}$ devices are PDMS based, since it exhibits a unique feature of permeability that allows for gas exchange within the outside atmosphere. Thus, PDMS permeability permits oxygen to enter the system, which greatly reduces the need for oxygen supply unit (oxygenator) [25]. Also, high surface to volume ratio experienced in $\mu \mathrm{F}$ devices contributes into an excess in oxy- 
gen supply and less molecular dilution [20]. Leclerc et al. [25] have estimated the oxygen consumption within a $\mu \mathrm{F}$ domain by the product of cells' density in the $\mu \mathrm{F}$ compartment per, the consumption of a single cell per second, and cell culture area.

\subsection{Anticipated objectives of the $\mu \mathrm{F}$ system}

In light of the relevant literature flourished in this paper and personal practical experiences in microfabrication and bio-experiments, it is a pre-requisite to have the culture chamber sufficiently large for cell to grow and differentiate, as well as to consider tie-in-connection constraints (i.e. syringe pump, disposal capillary tube, etc.). However, dense tie-in $\mu \mathrm{F}$ environment of mixers, valves, pumps and so forth, affects the throughput of the system by adding more mechanical constraints; this shall also deviate us from one of the main objectives in designing the LoC system, which is acquiring simplicity of the structure and experimental setup.

A uniform velocity profile is anticipated to ensure uniform distribution of nutrients (parabolic laminar flow profile). It is quite essential to design the channel breadth large enough to avoid adhesive cell accumulation (channel clogging). In order to prevent breakage of the glass-PDMS bonding, the channel has to be designed such that a pressure drop experienced within the channel is maintained at minimal during LoC system normal performance. An essential design aspect of a rectangular channel is the hydrodynamic resistance factor that is directly proportional to channel length, and inversely proportional to channel width and the third power of channel height [27]. From the biological perspective, osmotic pressure [35] is essential for cell viability, and it should be considered that many cells do require attachments to solid surfaces prior to proliferation process to commence [28].

Figure 3 summarizes the aforementioned factors flourished in this work, which are required to maintain cell viability within a confined environment such as that of a $\mu \mathrm{F}$ and MEA system. Incorporating all these factors in the proposed design of the bio-mechatronic system, introduced in sections 2.3 and 3, is beyond the objectives of extracting the electrophysiology and mechanics of cells within this research, upon which the experiments are anticipated to be conducted for a short/ moderate time period, through which cells' viability and integrity will be maintained and preserved. In the following sections, Computational Fluid Dynamics (CFD) and Finite Element Method (FEM) are implemented as part of the design analysis approach prior to a microfabrictaion and characterization process of $\mu \mathrm{F}$ and MEA system.

\subsection{System level design $(\mu \mathrm{F}$ flow $)$}

As part of the detailed engineering analyses of the $\mu \mathrm{F}$ system, and the approach of utilizing a pressure difference via a syringe at the inlet and outlet ports to move fluids by inducing driven flow pressure, a Computational Fluid Dynamic (CFD) analysis, COMSOL Multiphysics 4.2a (Burlington, MA, USA), is performed to visualize and predict flow characteristics of the PBS medium. The objective of this analysis is to allocate optimal dimensions of the system that would contribute in sufficiently controlling pressure, and eventually injecting and ejecting medium solution to and out of the system at a reasonable velocity rate. Furthermore, this first-hand empirical analysis will greatly assist in analysing the flushing process of PBS within the microsystem for several experimental uses, thus increasing system durability and usability.

In the $\mu \mathrm{F}$ system, the flow is laminar and experiences a no-slip condition at walls, thus leading to a parabolic flow profile and limiting flow rate, which eventually leads to dispersion of sample plugs [36]. Laminar flow avails ease of controllability of the system [20]. $\mu$ F system exhibits a high surface 


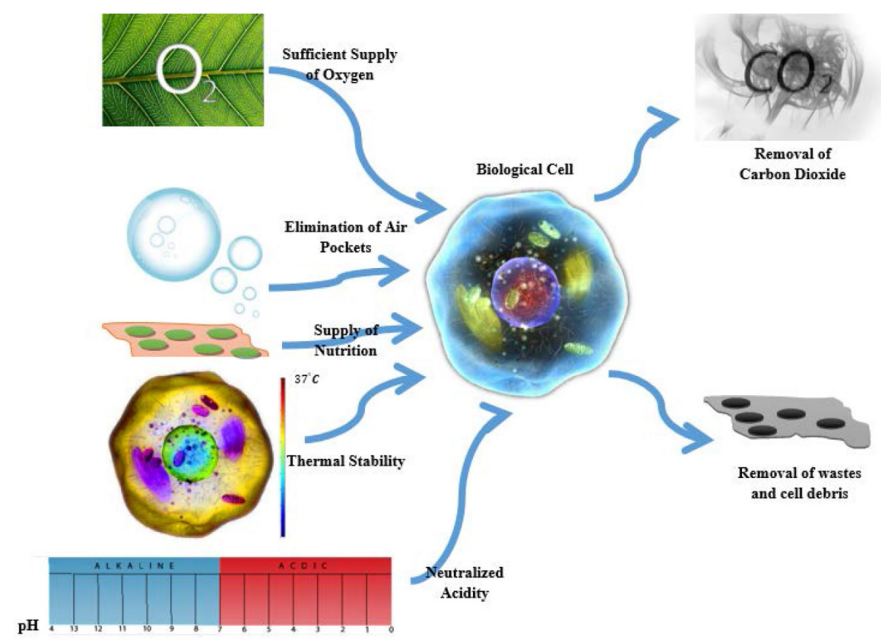

Figure 3: Requirements of cell viability by supplying sufficient oxygen rate and nutrition, elimination of air pockets, providing neutralized acidity and thermal stability environment and removal of wastes, insoluble cell debris and carbon dioxide from the flow system.

to volume ratio (low characteristic length of the system), indicating a highly viscous laminar flow based on low Reynolds' number definition [36]. Accordingly, for an incompressible flow, the inertia term of the Navier-Stokes equation drops out [37]. Another problematic design issue is flow resistance, which is found to be directly proportional to channel length and inversely proportional to its width [36]. The size of the $\mu \mathrm{F}$ device should be selected such that it would hold an adequate amount of cell media (biological constraint).

\subsection{1 $\mu \mathrm{F}$ model}

The $\mu \mathrm{F}$ system analysis has been performed in several iterations, where depth and width of channels, dynamic viscosity and density of the PBS solution, and applied pressures are all held fixed, while the length of the channel varies (all resultant iteration graphs are not shown). The density and dynamic viscosity of the PBS buffer medium are $1.06 \times 10^{3} \mathrm{Kg} / \mathrm{m}^{3}$, and $1.99 \times 10^{3} \mathrm{~Pa} . \mathrm{sec}$, respectively [38, 39]. The analysis is conducted by considering a time domain that will efficiently contribute in design optimization. Therefore, the differential pressure injected into the system via a syringe mechanism in the inlet reservoir with respect to the outlet is timely controlled. The pressure at the outlet reservoir is set to zero, where there will be no viscous stresses. Similarly, it is assumed that there will be no viscous stresses at pressure inlet exerted near the culturing reservoir upper edge, and it is defined in the following time-dependent equation:

$$
P_{\text {applied }}=P_{o}+P_{\mathrm{a}} \sin (w t)
$$

The pressures offset and amplitude are denoted as $P_{o}$ and $P_{\alpha}$, respectively, while applied pressure at the inlet is $P_{\text {applied }}$ The buffer medium flow within the $\mu \mathrm{F}$ system is laminar; thus, the flow pattern and characteristics are depicted by solving the incompressible Navier-Stokes, eqn (2), and continuity equation, eqn (3), numerically within a 4-second-time domain.

$$
\begin{gathered}
\rho \frac{\partial u}{\partial t}-\nabla \cdot \eta\left(\nabla u+(\nabla u)^{T}\right)+\rho u \cdot \nabla u+\nabla P=0 \\
\nabla \cdot u=0
\end{gathered}
$$




$$
\begin{gathered}
\left(\nabla \cdot \eta\left(\nabla u+(\nabla u)^{T}\right)\right) \cdot n=0 \\
p=p_{\text {applied }}
\end{gathered}
$$

where, $u$ is the velocity, $\rho$ is density, $\eta$ is the dynamic viscosity of the solution and $\eta$ is the outward normal unit vector of the boundary. A no-slip condition is imposed, where velocity components at the walls are zero. The solution maintains a uniform density, and viscous stress effect diminishes at the inlet and outlet of the $\mu \mathrm{F}$ system.

\subsubsection{CFD: $\mu$ F system characterization}

Figure 4A depicts the velocity-field distribution and pressure at walls throughout the $\mu \mathrm{F}$ system. The right and left level indicators at the right side of Fig. 4A, show pressure and velocity distribution, respectively. For pressure indicator, yellow indicates maximum pressure while dark red is minimum pressure experienced within the system. As for velocity distribution level indicator, red signifies maximum velocity, while blue is minimum. Fig. 4D indicates that the streamlines (arrow velocities), within the microfluidic channel, are maximum (squeezing) and experience a Gaussian velocity distribution across the width of the channel, where the maximum velocity is at the center of the channel. This result is anticipated as it depicts typical Poiseuille (laminar) flow.

From the obtained results, a large pressure drop (pressure loss) would reduce the flow in the microchannels, and thus affecting the flushing process for proper maintenance of the system. This obtained optimal CFD result predicts flow pattern, and helps designers to reach a system that exhibits a proper overall pressure distribution-achieving better flow controllability over time.

From the obtained results of the several empirical iterations (just optimal case is shown), it has proven that in a longer channel case, the flow is slow due to a very large pressure drop caused by frictional losses. It should be noted that the larger the pressure loss, the slower the flow in the channels. On the other hand, shorter channels experience high pressure in a compacted channel, and thus experience high velocities immediately preceding the outlet, which will add complexity in controlling the flow. However, the optimization process has yielded a system as shown in Fig. 4 with a reasonable proper pressure distribution along the channel, and thus has a reasonable velocity near the outlets and offers a reasonable flow control. In addition to the analyses, the resultant size of the $\mu \mathrm{F}$ device as

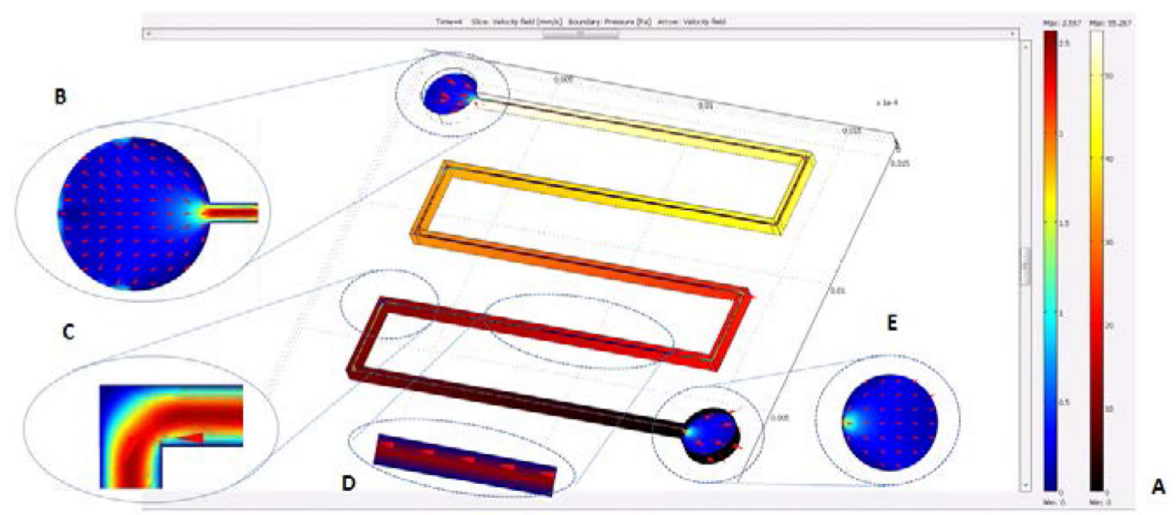

Figure 4: (A) Pressure at walls and velocity field in the microchannel at $t=4 \mathrm{sec}$, (B) Velocity profile distribution at the neck exit of the culturing reservoir and into the channel, (C) Built-up pressure at sharp edges, (D) Gaussian Poiseuille velocity profile within the microchannel and (E) Pressure at minimum and velocity distribution at the exit reservoir. 
depicted in Fig. 4 meets the constraint/requirement of holding an adequate amount of cell media. The channel width is optimally chosen to fairly encapsulate the width of cell-line growths within a confined environment. As per the empirical analyses, the channel width is $228 \mu \mathrm{m}$, and length varies on each side of the $\mu \mathrm{F}$ system. The diameter of the culturing and outlet reservoirs, where cell culturing is initiated and disposed form the system respectively, are of equal size of $1.77 \mathrm{~mm}$ - giving sufficient space for syringe tip to inject into a feeding tube that is connected to a pin adaptor/connector. Based on the flow visualization analyses the entire $\mu \mathrm{F}$ system has a uniform depth of $20 \mu \mathrm{m}$.

\section{INTEGRATED MULTI ELECTRODE ARRAYS (IMEAS)}

In addition to the mechanical pressure driven flow exhibited in the previous section, the objective is to increase the versatility of the bio-mechatronic platform by considering a second driving force of the flow for future extension of this work: electroosmotic/electrokinetic and electrophoresis. This is in line of the Constructal law in identifying the imperfection of a current existing design, and arriving at enhancement tactics to elevate the existing flow system design. Thus, on the contrary to pressure-driven flow, electroosmotic/electrokinetic are utilizing electric field to induce fluid dynamics, whereas electrophoresis is used to cause motion of particles within the fluid [31]. In the study of [31], electrophoresis illustrates the movement of biological particles by electric fields, in which DNA for instance migrates towards a positive electrode, whereas electroosmotic flow is the fluid flow from a positive to a negative electrode [31]. Thus, the proposed system shall have the flexibility to switch between a simply operated-pressure-driven flow to an electro-kinetic flow: switching from the former to the latter mechanism greatly enhances cell trapping and manipulation process, and most importantly overcomes the deficiency of the pressure-driven flow with the incorporation of the no-slip condition at the $\mu \mathrm{F}$ walls, leading to a parabolic velocity distribution, and thus non-uniform distribution of nutrients, whereas electrokinetic driven flow exhibits a relatively uniform velocity distribution.

This added feature would contribute into cell trapping, deformation, and manipulation under a presence of an electric field. This is accomplished through integrated multi-electrode arrays (iMEAs), within the LoC/ $\mu \mathrm{F}$ domain.

\subsection{Electric field cell manipulation}

There are two constant demands by bio-experimentalists working on cell manipulation, to optimally control the flow and report changes in a real-time response. Although it is beyond the scope of this research in utilizing electric means in manipulating cells, it is worth re-stating that the limitations of pressure driven flow are overcome by utilizing an electric filed to impose cell movements via electrophoresis mechanism, which is quite essential, and it is a very dense area of research. Furthermore, it is worth stating that there is a third mechanism in manipulating cells, which is beyond the scope of this work as well, and that is the optical tweezers approach [40].

Issadore et al. [41] have comprehensively illustrated a method of cell manipulation through electric field frequency (i.e. dielectrophoresis, magnetophoresis, electrofusion), within a hybrid $\mathrm{IC} / \mu \mathrm{F}$ chip. They have shown an ease of controllability of cell manipulation over a pico liter of volume. Their proposed system leads to a full automation in terms of deforming, trapping and moving biological cells at potentials in $\mathrm{MHz}$ frequency, whereas mixing and fusion are achieved by electrofusion and electroporation at potentials in a frequency range below $1 \mathrm{KHz}$. This is performed on the basis of dielectrophoresis (DEP) in a vast multi-electrode environment. Such system provides an automated multitask LoC platform that eliminates the bulky micromechanical tie-ins of the system, e.g. 
mixers, pumps, latches and valves. They have also illustrated in their experiments that cell-membrane potential is dependent on the frequency of the imposed electric field, and it remains unharmed at frequencies below $1 \mathrm{KHz}$ within a range of a utilized DEP. Furthermore, this shall open a new frontier in analysing cell-cell communication without physical contact among cells, which is usually performed through a cellular gap-junction mechanism-gap junction is a physical biochemical connection among cell network [18]. Moreover, iMEAs contribute into extracting cell electrophysiology characteristics, and hence upon which the correlation between cancer cell mechanical properties shall be drawn. Cell manipulation and flow driven by electrical means are beyond the scope of this study, rather the main focus is on utilizing iMEAs in extracting the cell-membrane potential. MEAs contribute into high throughput of the LoC platform in terms of cell manipulation. An electrode is acting as a sensor in a passive mode (recording electrode), and as a stimulator to the cell in an active mode.

\subsection{Capturing electrophysiology of cells}

It has been reported that the electrophysiology/electrochemical characteristics of even a very diminutive building component of a cell such as a protein have been detected by utilizing MEAs [8]. Therefore, electrodes are utilized for electrochemical detection $[6,42]$, where such electrochemical changes at a molecular level contribute into the bio-signalling process [43].

On the same application, Daworak and Wheeler [44] have designed and fabricated a novel and highly stable MEA integrated within PDMS microtunnels to capture the speed and direction of cellmembrane action potential propagation, which was on the magnitude of a $300 \mu \mathrm{V}$ electric signal of isolated axon cells; they have developed a system consisting of four culturing wells and one central interconnected by microtunnel arrays, where three configurations of varying $\mathrm{Au} / \mathrm{Cr}$ microelectrode width $(25 \mu \mathrm{m}, 50 \mu \mathrm{m}$, and $75 \mu \mathrm{m})$, with a pitch distance of $200 \mu \mathrm{m}$ have been placed underneath the PDMS channel to investigate the dependence of signal amplitude on electrode size. The 50 and $75 \mu \mathrm{m}$ electrodes produce larger signals depending on their configuration and size within the channel. The theory behind having the MEA buried beneath the PDMS channel floor is to create a high amplification of the induced potential considering that the micro-size of the tunnel experiences a very high resistivity of $16 \mathrm{M} \Omega$ [44].

On an instrumentation aspect, current to voltage (I/V) convertor and a system of digital-to-analog convertors (DACs) are utilized to assure ease of controllability of induced potentials through gold patterned electrodes [45].

\subsection{MEA configuration}

An overlap of neighbouring electrodes could affect the performance of the entire MEA system. Freire et al. [46] have investigated the influence of the number of electrodes, their distribution pattern on the basis of gain of the signal-to-noise ratio (SNR) and overlap (cross talk) between adjacent electrodes due to diffusion layers. Based on their previous studies, the minimum maintained distance between two neighbouring electrodes in MEA should be greater than six times their diameter of wire-shaped electrodes to eliminate overlap of diffusion layers. Also, they have investigated different variations of distances between electrodes on detection limit; the faradic current increases with distance, which negatively affect the detection limit. For their particular experiment, it is found that the distances between electrodes should be of 20 times the electrode diameter; also the detection limit resolution weakens, affecting the overall SNR of the system. Usually, the range of distance between two neighbouring electrodes varies from 1-10 $\mu \mathrm{m}$; however, it is subject to microfabrica- 
tion constraints as well as the main desire of achieving high sensitivity by having a strong electric field [42]. A reduction in electrode surface leads to a reduction in obtained signals [30].

\subsection{System level design and characterization: MEA}

As for the MEA configuration, important findings for the design constraints associated with electrode cross talk, uniform stimulation threshold, heating of cell membranes and electrode erosion have been extensively investigated and discussed by Planker et al. [47]. Their conclusion is that large-electrode cell displacement leads to a cross talk between adjacent electrodes, and thus high charge density and power stimulation-eventually introducing heat to the system and causing electrodes to be eroded (poor resolution output). They have proven that the stimulating-threshold current is directly proportional to electrode-cell displacement as well as electrode size. As a result of their findings, the optimal distance between neighbouring electrodes should preserve a tenth of an electric field of one electrode to a neighbouring recording one.

In this research, a three-dimensional finite-element-method (FEM) model of two-upper-and-lower neighbouring electrodes within a $\mu \mathrm{F}$ channel has been constructed, where the width of the electrode is held fixed at $228 \mu \mathrm{m}$ (comparable to channel width), while the pitch distance between the stimulating and recording electrode was varied through several iterations. The electrodes used in the simulation are gold coated and rectangular shaped. The objective of the model is to visualize the current density distribution within the PBS buffer medium, and thus identify an optimal distance that provides a uniform current density distribution, and allows sufficient space for cell-line growth between two neighbouring electrodes.

The principal physical relations are based on Maxwell's continuity eqn (6), and two constitutive relations interrelating electric field, total current density, and electric potential of the system (7) and (8):

$$
\begin{gathered}
-\nabla \cdot(\sigma \nabla V)=0 \\
E=-\Delta V \\
J=\sigma E
\end{gathered}
$$

where, $E$ is the electrical filed, $V$ is the potential, $J$ is current density, and $\sigma$ is conductivity. The conductivity of the PBS and gold electrode are $179 \times 10^{-3} \frac{\mathrm{S}}{\mathrm{m}}$, and $45.2 \times 10^{6} \frac{\mathrm{S}}{\mathrm{m}}$, respectively $[48,49]$.

Figure 5 shows the potential profile on electrodes as well as arrow representation of a total current density within the microchannel; the level indicator represents potential experienced within the system and arrows illustrate current distribution between the active and passive electrode. After several iterations in COMSOL Multiphysics 4.2a, the current density distribution is relatively uniform at a pitch distance of $20 \mu \mathrm{m}$. At this distance, the stimulating electrode doesn't experience comparatively high current density at its edges. High current density leads to a biofluidic coagulation, which will eventually affect the efficiency of cell electrophysiology measurements. Furthermore, reaching a uniform current distribution is desirable in designing an optimal distance between electrodes on the essence of providing stability of measurements over an extended period of time, and ensuring a damage-free cell membrane. Remarkably, the obtained optimal distance between the two neighbouring electrodes that yielded a uniform total current distribution is in harmony of the obtained channel depth of the microfluidic system in achieving a proper pressure and velocity distribution: enabling better flow controllability (flow system integrity). The obtained optimal depth also complies with a biological constraint of entrapping cells, whose diameters are on average of 8-10 $\mu \mathrm{m}$ [6]. 


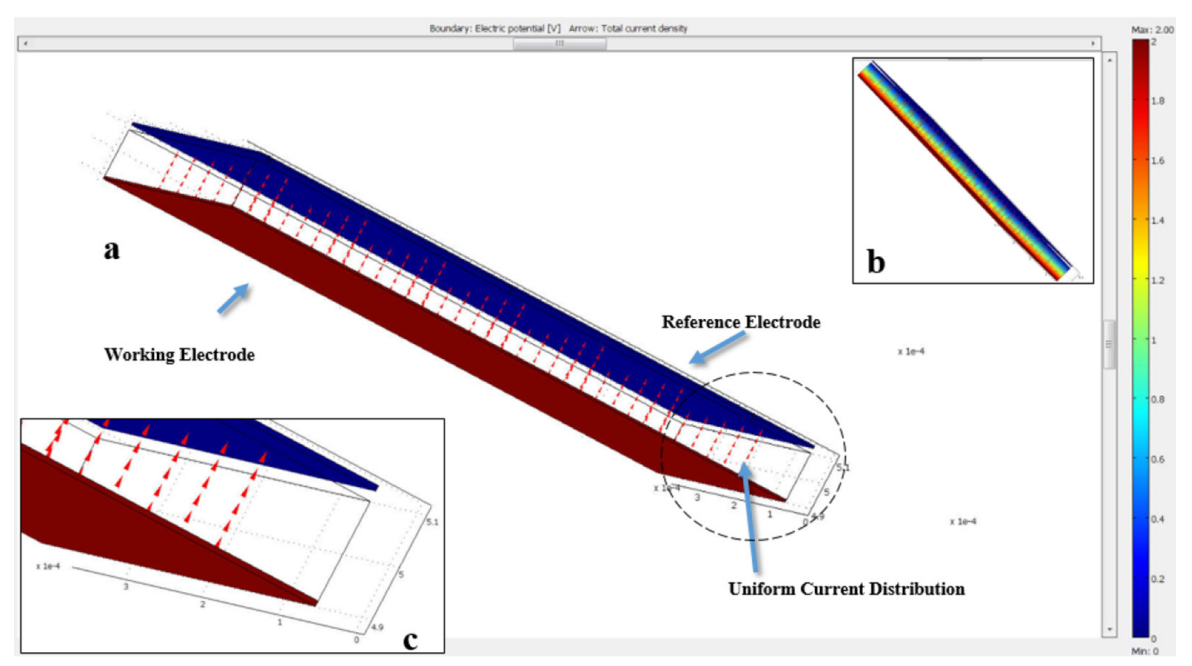

Figure 5: (a) Empirical analysis of a stimulated working electrode and recording reference electrode, (b) potential gradient distribution within the electrical field between working and reference electrode and (c) enlargement of the small dotted circled section showing a uniform current distribution between the two electrodes within a $\mu \mathrm{F}$ channel.

\section{CONCLUSION}

This work has highlighted the dominant criteria for cell viability: sufficient supply of oxygen and nutrition, stabilized thermal system and $\mathrm{pH}$ rate, elimination of air pockets, and disposal of carbon dioxide, cell debris and wastes. Furthermore, this paper has shed light on extraction of cell electrophysiology, and has manifested the importance of in vitro testing as opposed to in vivo and petri-dish culturing. It is clearly illustrated how nature defines the engineering requirements of two important building blocks of LoC module- $\mu \mathrm{F}$ and MEAs. The understanding of cellular biology requirements as a flow system defines a design strategy, on the basis of science, where spatial and temporal pattern, as well as system configuration of the major building components of $\mathrm{LoC}$ ( $\mu \mathrm{F}$ and MEAs) are prominent. Such tactic shall greatly save time, resources, and it shall avoid trial and error during microfabricartion, and preserve efforts and provide a solid platform of understanding the laying physics when conducting in vitro experiments.

The design synthesis, in light of biological constraints, has yielded empirical analyses in terms of visualizing the flow within a $\mu \mathrm{F}$ domain that ensures uniform pressure gradient distribution, and uniform flow rate: ensuring controllability in flushing the system with PBS medium. This is accomplished through the constitutive relation sets forward in Navier Stokes and continuity equations. From the MEA perspective, the FEM optimal result has illustrated a uniform current distribution between two adjacent electrodes. Such design syntheses on the essence of analysing cellular viability, and design analyses of the CFD and FEM models should greatly assist designers prior to microfabricating $\mathrm{i} \mu \mathrm{F}$ and iMEAs LoC system; such flourished design syntheses and analyses within this work have greatly contributed in microfabricating the LoC shown in Fig. 6. The microfabrication and in vitro experiments shall be further addressed in future works. 


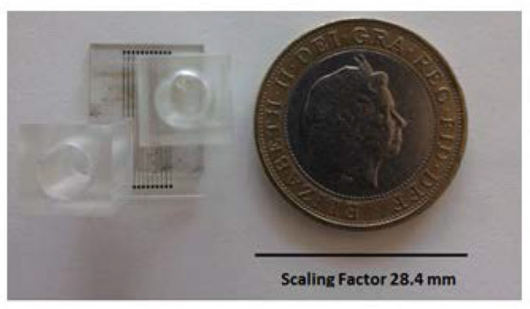

A

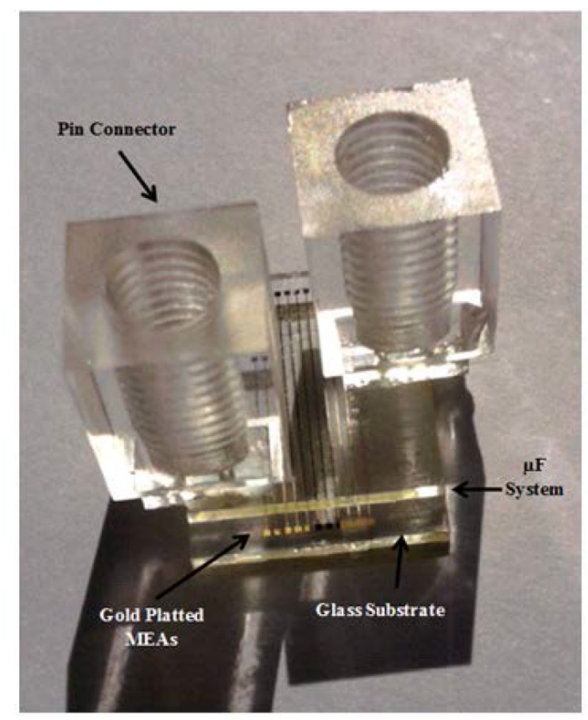

Figure 6: (A) A top view of the LoC module with two-Sterling British Pounds as a scaling factor (B) An image showing connecting pins of culturing and outlet reservoir, and gold platted upper and lower MEAs patterned on two glass substrates (upper and lower) which are crossing the microfluidic channels.

\section{REFERENCES}

[1] Skelley, A.M., Cleaves, H.J., Jayarajah, C.N., Bada, J.L. \& Mathies, R.A., Application of the mars organic analyzer to nuceleobase and amine biomarker detection. Astrobiology, 6(6), pp. 824-837, 2006. doi: http://dx.doi.org/10.1089/ast.2006.6.824

[2] Sims, C.E. \& Allbritton, N.L., Analysis of single mammalian cells on-chip. Lab Chip, 7, pp. 423-440, 2007. doi: http://dx.doi.org/10.1039/b615235j

[3] Seidl, J., Knuechel, R. \& Kunz-Schughart, L.A., Cytometry, 36, pp. 102-111, 1999. doi: http:// dx.doi.org/10.1002/(sici)1097-0320(19990601)36:2\%3C102::aid-cyto3\%3E3.3.co;2-4

[4] Bejan, A. \& Lorente, S., Design with Constructal Theory, Hoboken: Wiley, 2008. doi: http:// dx.doi.org/10.1002/9780470432709

[5] Bejan, A. \& Zane, J.P., Design in Nature, Doubleday: New York, 2012.

[6] Alqabandi, J., Abdul Mottal, U. \& Youcef-Toumi, K., Extracting cancer cell line electrochemical parameters at the single cell level using a microfabricated device. Biotechnology Journal, 4, pp. 216-223, 2009. doi: http://dx.doi.org/10.1002/biot.200800321

[7] Breslauer, D.N., Lee, P.J. \& Lee, L.P., Microfluidics-based systems biology. Mol. BioSyst., 2, pp. 97-112, 2006. doi: http://dx.doi.org/10.1039/b515632g

[8] Fragoso, et al., Integrated microfluidic platform for the electrochemical detection of breast cancer markers in patient serum samples. Lab Chip, 11, pp. 625-631, 2011. doi: http://dx.doi. org/10.1039/c0lc00398k

[9] Kim, L., Toh, Y.-C., Voldman, J. \& Yu, H., A practical guide to microfluidic perfusion culture of adherent mammalian cells. Lab Chip, 7, pp. 681-694, 2007. doi: http://dx.doi.org/10.1039/b704602b

[10] Hung, P.J., Lee, P.J., Sabounchi, P., Aghdam, N., Lin, R. \& Lee, L.P., A novel high aspect ratio microfluidic design to provide a stable and uniform microenvironment for cell growth in a high throughput mammalian cell culture array. Lab Chip, 5, pp. 44-48, 2005. doi: http://dx.doi. org/10.1039/b410743h 
[11] Kim, L., Vahey, M.D., Lee, H.-Y. \& Voldman, J., Microfluidic arrays for logarithmically perfused embryonic stem cell culture. Lab Chip, 6, pp. 394-406, 2006. doi: http://dx.doi. $\underline{\operatorname{org} / 10.1039 / \mathrm{b} 511718 \mathrm{f}}$

[12] Nevill, J.T., Cooper, R., Dueck, M., Breslauer, D.N. \& Lee, L.P., Integrated microfluidic cell culture and lysis on a chip, Lab Chip, 7, pp. 1689-1695, 2007. doi: http://dx.doi.org/10.1039/b711874k

[13] Dimov, I.K., Kijanka, G., Park, Y., Ducre, J., Kang, T. \& Lee, L.P., Integrated microfluidic array plate (iMAP) for cellular and molecular analysis. Lab Chip, 11, pp. 2701-2710, 2011. doi: http://dx.doi.org/10.1039/c1lc20105k

[14] Escámez M.J., García M., Larcher F., Meana A., Muñoz E., Jorcano J.L. \& Del Río M., An In Vivo Model of Wound Healing in Genetically Modified Skin-Humanized Mice. Journal of Investigative Dermatology, 123, pp. 1182-1191, 2004. doi: http://dx.doi.org/10.1111/j.0022202x.2004.23473.x

[15] Shultz, L.D., Ishikawa, F. \& Greiner, D.L., Humanized mice in translational biomedical research. Nature Reviews Immunology, 7, pp. 118-130, 2007. doi: http://dx.doi.org/10.1038/nri2017

[16] Vickerman, V., Blundo, J., Chung, S. \& Kamm, R., Design, fabrication and implementation of a novel multi-parameter control microfluidic platform for three-dimensional cell culture and real-time imaging, Lab Chip, 8, pp. 1468-1477, 2008. doi: http://dx.doi.org/10.1039/b802395f

[17] Gmez-Sjberg, R., Leyrat, A.A., Pirone, D.M., Chen, C.S. \& Quake, S. R., Versatile Fully Automated, Microfluidic Cell Culture System. Anal. Chem., 79, pp. 8557-8563, 2007. doi: http:// dx.doi.org/10.1021/ac071311w

[18] Meyvantsson, I. \& Beebe, D.J., Cell Culture Models in Microfluidic Systems. Annu. Rev. Anal. Chem., 1, pp. 423-449, 2008. doi: http://dx.doi.org/10.1146/annurev.anchem.1.031207.113042

[19] El-Ali, J., Sorger, P.K. \& Jensen, K.F., Cells on chips. Nature, 442, pp. 403-411, 2006. doi: http://dx.doi.org/10.1038/nature05063

[20] Zilkowska, K., Kwapiszewski, R. \& Brzzka, Z., Microfluidic devices as tools for mimicking the in vivo environment. New J. Chem., 35, pp. 979-990, 2011. doi: http://dx.doi.org/10.1039/ c0nj00709a

[21] Dorland's Medical Dictionary for Health Consumers. Saunders an imprint of Elsevier, Inc., 2007.

[22] Rhee, S.W., Taylor, A.M., Tu, C.H., Cribbs, D.H., Cotman, C.W. \& Jeon, N.L., Patterened cell culture inside microfluidic devices. Lab Chip, 5, pp. 102-107, 2005. doi: http://dx.doi. org/10.1039/b403091e

[23] Kim, C., Lee, K.S., Bang, J.H., Kim, Y.E., Kim, M.C., Oh, K.W., Lee, S.H. \& Kang, J.Y., 3-Dimensional cell culture for on-chip differentiation of stem cells in embryoid body. Lab Chip, 11, pp. 874-882, 2011. doi: http://dx.doi.org/10.1039/c0lc00516a

[24] Romer, L.H., Konstantin, G.B. \& Garcia, J.G.N., Circ. Res., 98, pp. 606-616, 2006.

[25] Lecrec, E., Sakai, Y., \& Fujii, T., Cell Culture in 3-Dimensional Microfluidic Structure of PDMS (polydimethylsiloxane), Biomedical Microdevices, 5(2), pp. 109-114, 2003.

[26] Cheng, Y., Luo, X., Tsao, C.-T., Wu, H.-C., Betz, J., Payne, G.F., Bentley, W.E. \& Rubloff, G.W., Biocompatible multi-address 3D cell assembly in microfluidic devices using spatially programmable gel formation. Lab Chip, 11, pp. 2316-2318, 2011. doi: http://dx.doi. org/10.1039/c1lc20306a

[27] Stone, H.A., Introduction to Fluid Dynamics for Microfluidic Flows, CMOS Biotechnology: Springer, pp. 5-30, 2007. doi: http://dx.doi.org/10.1007/978-0-387-68913-5 2

[28] Prakash, S.B. \& Abshire, P., Tracking cancer cell proliferation on a CMOS capacitance sensor chip. Biosensors and Bioelectronics, 23, pp. 1449-1457, 2008. doi: http://dx.doi.org/10.1016/j. bios.2007.12.015

[29] Eddington, D.T., Liu, R.H., Moore, J.S. \& Beebe, D.J., An organic self-regulating microfluidic system. Lab Chip, 1, pp. 96-99, 2001. doi: http://dx.doi.org/10.1039/b108078d 
[30] Estrela, P., \& Miglirato, P., Chemcial and biological sensors using polycrystalline silicon TFTs. J. Mater. Chem., 17, pp. 219-224, 2007. doi: http://dx.doi.org/10.1039/b612469k

[31] Cross, J.D. \& Craighead, H.G., Micro-and Nanofluidics for Biological separations, CMOS Biotechnology: Springer, pp. 31-75, 2007. doi: http://dx.doi.org/10.1007/978-0-387-68913-5 3

[32] Ezashi, T., Das, P. \& Roberts, R.M., Proc. Natl. Acad. Sci. U. S. A., 102, pp. 4783-4788, 2005. doi: http://dx.doi.org/10.1073/pnas.0501283102

[33] Allen, J.W., Khetani, S.R., \& Bhatia, S.N., Toxicol. Sci., 84, pp. 110-119, 2005.

[34] Tilles, A.W., Baskaran, H., Roy, P., Yarmush, M.L. \& Toner, M., Biotechnol. Bioeng., 73, pp. 379-389, 2001. doi: http://dx.doi.org/10.1002/bit.1071

[35] Lee, K.L., Baker, R.W. \& Lonsdale, H.K., Membranes for power generation by pressureretarded osmosis. Journal of Membrane Science, Elsevier B.V., 8(2), pp. 141-171, 1981. doi: http://dx.doi.org/10.1016/s0376-7388(00)82088-8

[36] Seger, U., Electrical Cell Manipulation in Microfluidic Systems, Ph.D. Dissertation, Ecole Polytechnique Fereale Lausanne, EPFL 2006.

[37] Body, J.P., Yager, P., Goldstein, R.E. \& Austin, R.H., Biotechnology at low Reynolds numbers. Biophysical Journal, 71, pp. 3430-3441, 1996. doi: http://dx.doi.org/10.1016/s00063495(96)79538-3

[38] Basalo, I., Chahine, N., Kaplun, M., Chen, F., Hung, T. \& Ateshian, G., Chondrotini sulfate reduces the friction coefficient of articular cartilage. Journal of Biomechanics, 40, pp. 1847 1854, 2007. doi: http://dx.doi.org/10.1016/j.jbiomech.2006.07.007

[39] Oren, A., Pri-El, N, Shapiro, O. \& Siboni, N., Buoyancy studiesin natural communities of square gas-vacuolate archea in saltern crystallizer ponds. Saline Systems, 2(4), 2006.

[40] Hellmich, W., Greif, D., Palargus, C., Anselmetti, D. \& Ros, A.J., Chromatogr, A., 1130, pp. 195-200, 2006. doi: http://dx.doi.org/10.1016/j.chroma.2006.06.008

[41] Issadore, D., Franke, T., Brown, K.A., \& Westervelt, R.M., A microfluidic microprocessor: controlling biomimetic containers and cells using hybrid integrated circuit/microfluidic chips. Lab Chip, 10, pp. 2937-2943, 2010. doi: http://dx.doi.org/10.1039/c01c00092b

[42] Lisdat, F., \& Schafer, D., The use of electrochemical impedance spectroscopy for biosensing. Anal. Bioanal. Chem., 391, pp. 1555-1567, 2008. doi: http://dx.doi.org/10.1007/s00216-008$\underline{1970-7}$

[43] Ficher, N.O., Tarasow, T.M. \& Tok, J.B.-H., Heightened sense for sensing: recent advances in pathogen immunoassay sensing platforms. Analyst, 132, pp. 187-191, 2007. doi: http://dx.doi. org/10.1039/b615477h

[44] Dworak, B.J. \& Wheeler, B.C., Novel MEA platform with PDMS microtunnels enables the detection of action potential propagation from isolated axons in culture. Lab Chip, 9, pp. 404 410, 2009. doi: http://dx.doi.org/10.1039/b806689b

[45] Rohwedder, J.J.R. \& Pasquini, C., Multi-electrode detection in voltammetry Part I. A versatile multi-channel voltammetric instrument. Analyst, 123, pp. 1641-1648, 1998. doi: http://dx.doi. org/10.1039/a801101j

[46] Freire, R.S., Rohwedder, J.J.R. \& Pasquini, C., Multi-electrode detection in voltammetry Part 3. Effects of array configuration on the Hadamard multiplexed voltammetric technique. Analyst, 124, pp. 1657-1660, 1999. doi: http://dx.doi.org/10.1039/a904670d

[47] Planker, D., Vankov, A., Huie, P. \& Baccus, S., Design of a high-resolution optoelectronic retinal prosthesis. Journal of Neural Engineering, 2005. doi: http://dx.doi.org/10.1088/17412560/2/1/012

[48] Johnson, A., Sadoway, D., Cima, M. \& Langer, R., Design and Testing of an Impedance-Based Sensor for Monitoring Drug Delivery. Journal of the Electrochemical Society, 2005. doi: http:// dx.doi.org/10.1149/1.1824045

[49] Environmental, Chemistry \& Hazardous Materials News, Careers \& Resources: http://environmentalchemistry.com. 